\title{
A new Markov Binomial distribution
}

\author{
Leda D. Minkova
}

Edward Omey

HUB RESEARCH PAPERS 2011/24

ECONOMICS \& MANAGEMENT

NOVEMBER 2011
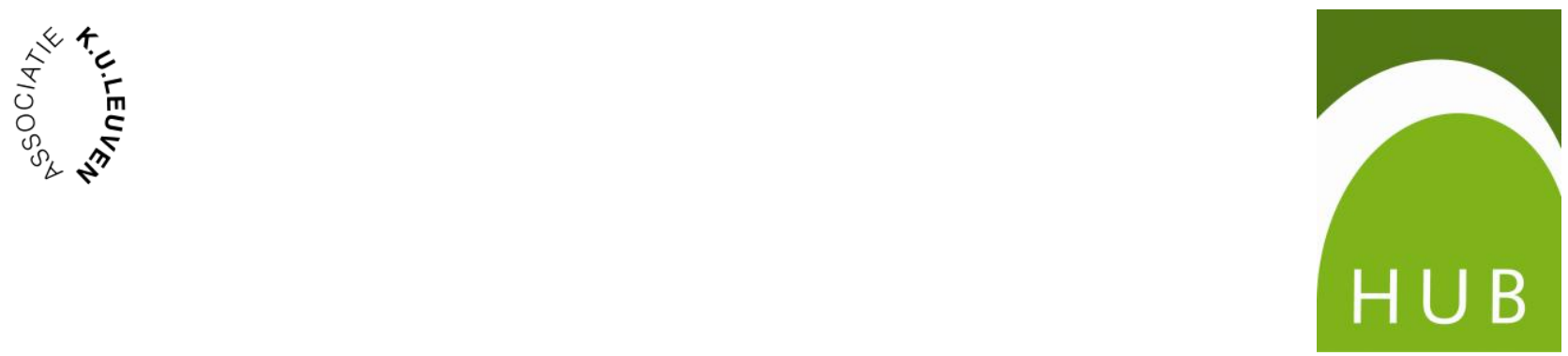


\title{
A new Markov Binomial distribution
}

\author{
Leda D. Minkova ${ }^{1}$ and Edward Omey ${ }^{2}$ \\ ${ }^{1}$ Faculty of Mathematics and Informatics \\ Sofia University "St. Kl. Ohridski" \\ e-mail: leda@fmi.uni-sofia.bg \\ ${ }^{2}$ HUB, Stormstraat 2, 1000 Brussel \\ e-mail: edward.omey@hubrussel.be.
}

\begin{abstract}
In this paper, we introduce a two state homogeneous Markov chain and define a geometric distribution related to this Markov chain. We define also the negative binomial distribution similar to the classical case and call it NB related to interrupted Markov chain. The new binomial distribution is related to the interrupted Markov chain. Some characterization properties of the Geometric distributions are given. Recursion formulas and probability mass functions for the NB distribution and the new binomial distribution are derived.
\end{abstract}

Key words: Homogeneous Markov chain, Interrupted Markov chain, Geometric distribution related to Markov chain, Inflated negative binomial distribution, New binomial distribution.

AMS subject classifications: 60E05; 62P05.

\section{Introduction}

It is known that many basic counting distributions are defined on a sequence of independent and identically distributed Bernoulli random variables. Many other distributions are defined by compounding and mixing. Another way of obtaining new discrete distributions is to define the counting distributions related to some Markov chain, see Viveros et al. (1994) and Omey et al. (2008). Assuming some dependency in the sequence of Bernoulli variables, gives an additional parameter, and could be a more realistic model in practice, see Omey and Van Gulck (2006).

In this paper we introduce some discrete distributions related to two state homogeneous Markov chain. In Section 2, we introduce a homogeneous Markov chain, defined on the sequence of Bernoulli variables. The additional parameter in the Markov chain is interpreted 
as a correlation coefficient. We also define a geometric distribution related to this Markov chain. We give some useful properties and characterizations. In Section 3, we define an interrupted Markov chain and a corresponding negative binomial distribution, called inflated negative binomial. We derive the probability mass function (PMF), recursion formulas, central limit theorem, and a limiting distribution. In Section 4, we use an extension of the well known relations between negative binomial and binomial distribution and define a binomial distribution related to the interrupted Markov chain. This distribution is called a new binomial distribution. Some properties and recursions are given.

\section{A geometric distribution related to a Markov Chain}

Consider a sequence of binary random variables $\left(X_{n}, n=1,2, \ldots\right)$, where the states 1 and 0 appear with stationary probabilities

$$
P\left(X_{n}=1\right)=\pi \text { and } P\left(X_{n}=0\right)=q=1-\pi .
$$

We call the state 1 a "success" and the state 0 a "failure". We assume that the $X_{n}$ form a Markov chain with transition probabilities

$$
\begin{aligned}
& P\left(X_{n+1}=1 \mid X_{n}=1\right)=1-q(1-\rho) ; \\
& P\left(X_{n+1}=0 \mid X_{n}=0\right)=1-\pi(1-\rho),
\end{aligned}
$$

where $\rho \in[0,1)$ and $n=1,2, \ldots$ The transition matrix is given by

$$
\mathbf{P}=\left(\begin{array}{cc}
1-\pi(1-\rho) & \pi(1-\rho) \\
q(1-\rho) & 1-q(1-\rho)
\end{array}\right)
$$

The correlation coefficient is $\rho\left(X_{n}, X_{n+1}\right)=\rho$, for $n=1,2, \ldots$ and then the $n$ step transition matrix is given by

$$
\mathbf{P}^{n}=\left(\begin{array}{cc}
1-\pi\left(1-\rho^{n}\right) & \pi\left(1-\rho^{n}\right) \\
q\left(1-\rho^{n}\right) & 1-q\left(1-\rho^{n}\right)
\end{array}\right),
$$

see Feller (1971). This Markov chain represents a sequence of correlated Bernoulli random variables.

\subsection{Definitions}

Starting from $X_{1}, X_{2}, \ldots$, we define the random variables $Y$ and $Y_{S}$ as follows. The r.v. $Y$ counts the number of failures in the Markov chain up to the first success. The r.v. $Y_{S}$ counts the number of transitions up to the first success. Clearly we have $Y_{S}=Y+1$. It is clear that the PMF of $Y$ is given by

$$
\begin{aligned}
& P(Y=0)=\pi, \\
& P(Y=k) \quad=q(1-\pi(1-\rho))^{k-1} \pi(1-\rho), k=1,2, \ldots .
\end{aligned}
$$


The probability generating function (PGF) is given by

$$
\psi_{Y}(t)=E\left(t^{Y}\right)=\frac{\pi(1-\rho t)}{1-(1-\pi(1-\rho)) t}
$$

Note that the tail of $Y$ is given by

$$
P(Y \geq n)=q(1-\pi(1-\rho))^{n-1}, n=1,2, \ldots
$$

It is easy to verify that

$$
E(Y)=\frac{1-\pi}{\pi(1-\rho)} \quad \text { and } \quad \operatorname{Var}(Y)=\frac{E(Y)}{\pi(1-\rho)}+\frac{\rho}{\pi(1-\rho)^{2}}
$$

Definition 2.1 The r.v. Y defined by (2) and (3) is said to be geometrically distributed related to the Markov chain.

We use the notation $Y \sim I G e(\pi, \rho)$.

Remark 2.1 The distribution of $Y$ coincides with the inflated-parameter geometric distribution as in Minkova (2001, 2002). In Omey et al. (2008), this PMF was studied for general transition matrices. If $\rho=0$, then IGe $(\pi, \rho)$ corresponds to the usual geometric distribution.

Remark 2.2 We can also consider $-1<\rho<0$ as long as $(1-\pi)(1-\rho)<1$ and $\pi(1-\rho)<1$, i.e.

$$
\frac{-\rho}{1-\rho}<\pi<\frac{1}{1-\rho}
$$

\subsection{Characterizations of the $I G e(\pi, \rho)$}

We have the following result.

Theorem 2.1 (i) Let $Y$ be a non-negative, integer-valued random variable satisfying $P(Y=$ $0)=\pi$, where $\pi \in(0,1)$ and let $\rho \in[0,1)$. Then $Y \sim I G e(\pi, \rho)$ if and only if

$$
(1-\pi) P(Y \geq k+l)=(1-\pi(1-\rho)) P(Y \geq k) P(Y \geq l)
$$

for every $k, l=1,2, \ldots$

(ii) Let $Y$ be a non-negative, integer-valued random variable satisfying

$$
P(Y=0)=\pi \text { and } P(Y=1)=(1-\pi) \pi(1-\rho),
$$

where $\pi \in(0,1)$ and $\rho \in[0,1)$. Then $Y \sim \operatorname{IGe}(\pi, \rho)$ if and only if

$$
(1-\pi) P(Y>k+l)=P(Y>k) P(Y>l),
$$

for every $k, l=1,2, \ldots$ 
Proof. (i) If (2) holds, then (4) shows that (5) holds. Conversely, suppose that (5) holds. Using $P(Y \geq 1)=1-\pi$ we have

$$
(1-\pi) P(Y \geq k+1)=u(1-\pi) P(Y \geq k), k \geq 1,
$$

where $u=1-\pi(1-\rho)$. It follows that

$$
P(Y \geq k+1)=u P(Y \geq k)
$$

so that

$$
P(Y \geq k)=u^{k-1} P(Y \geq 1)=(1-\pi) u^{k-1} .
$$

This is (5).

(ii) From (4) we have that

$$
P(Y>n)=q(1-\pi(1-\rho))^{n}, n=1,2, \ldots,
$$

where $q=1-\pi$, and then (7) follows. Conversely, if (7) holds, then

$$
\begin{aligned}
q P(Y>n+1) & =P(Y>n) P(Y>1) \\
& =P(Y>n)(1-\pi-(1-\pi) \pi(1-\rho)) \\
& =q u P(Y>n),
\end{aligned}
$$

where $u=1-\pi(1-\rho)$. It follows that

$$
P(Y>n+1)=u P(Y>n), n=1,2, \ldots,
$$

and again (5) follows.

Theorem 2.2 Suppose that $X$ is a r.v. with $P(X \leq x)=1-\theta e^{-\lambda x}, \quad x \geq 0, \quad \lambda>0, \quad 0<$ $\theta<1$. Let $|X|=k$ if and only if $k-1<X \leq k, k \geq 0$. Then $|X|$ has an $\operatorname{IGe}(\pi, \rho)$ distribution with $\pi=1-\theta$ and $\rho=\left(e^{-\lambda}-\theta\right) /(1-\theta)$.

Proof. For $k=0$ we have

$$
P(|X|=0)=P(X \leq 0)=1-\theta .
$$

For $k \geq 1$, we have

$$
\begin{aligned}
P(|X|=k) & =P(k-1<X \leq k) \\
& =\theta e^{-\lambda(k-1)}-\theta e^{-\lambda k} \\
& =\theta\left(e^{-\lambda}\right)^{k-1}\left(1-e^{-\lambda}\right) .
\end{aligned}
$$

Comparing with $(2)$, we have $\pi=1-\theta$ and $(1-\pi) \pi(1-\rho)=\theta\left(1-e^{-\lambda}\right)$. It follows that

$$
1-\rho=\frac{1-e^{-\lambda}}{\pi}
$$


and then also that

$$
\rho=1-\frac{1-e^{-\lambda}}{\pi}=\frac{\pi-1+e^{-\lambda}}{\pi}=\frac{e^{-\lambda}-\theta}{1-\theta} .
$$

Clearly we have $1-\pi(1-\rho)=e^{-\lambda}$ as required.

Remark 2.3 If $\theta=e^{-\lambda}$, then $P(X \leq x)=1-e^{-\lambda(1+x)}$ and $X+1 \sim E X P(\lambda)$. The relation between an exponential distribution and the geometric distribution is well known, see Johnson et al. (2005).

Remark 2.4 If $\theta=1$, then $P(X \leq x)=1-e^{-\lambda x}$ and $X \sim E X P(\lambda)$, see Deng and Chhikara (1990), for example. Now we find that

$$
\begin{aligned}
& P(|X|=0)=0, \\
& P(|X|=k)=\left(e^{-\lambda}\right)^{k-1}\left(1-e^{-\lambda}\right), \quad k \geq 1 .
\end{aligned}
$$

\section{Inflated Negative Binomial Distribution}

\subsection{Definition}

Let $Y_{1}, Y_{2}, \ldots, Y_{r}$ denote independent r.v. with the same $I G e(\pi, \rho)$ distribution, and let $N(r)=Y_{1}+Y_{2}+\cdots+Y_{r}$. The distribution of $N(r)$ is called an inflated negative binomial distribution with parameters $\pi>0, \rho>0$ and $r \in \mathbb{N}_{0}$. Notation $N(r) \sim I N B(\pi, \rho, r)$.

The PGF of $N(r)$ is given by

$$
\psi_{N(r)}(t)=\left(\frac{\pi(1-\rho t)}{1-(1-\pi(1-\rho)) t}\right)^{r} .
$$

The random variables $Y_{i}$ are assumed to be independent. As a consequence, when a success appears, the Markov chain starts again at the origin and the initial PMF is given by $P\left(X_{1}=\right.$ $1)=\pi, P\left(X_{1}=0\right)=1-\pi$. Let the sequence $\left(X_{n}\right)$ denote a Markov chain with the following transition matrix $\mathbf{P}^{*}$ :

$$
\mathbf{P}^{*}=\left(\begin{array}{cc}
1-\pi(1-\rho) & \pi(1-\rho) \\
1-\pi & \pi
\end{array}\right) .
$$

This Markov chain will be called an interrupted Markov chain. The r.v. $N(r)$ counts the number of failures up to the $r$ th success in this Markov chain, see Omey et al. (2008), section 2.3 .

The $n$ step transition matrix is given by

$$
\mathbf{P}^{* n}=\left(\begin{array}{cc}
1-\frac{\pi(1-\rho)}{1-\pi \rho}\left(1-(\pi \rho)^{n}\right) & \frac{\pi(1-\rho)}{1-\pi \rho}\left(1-(\pi \rho)^{n}\right) \\
\frac{1-\pi}{1-\pi \rho}\left(1-(\pi \rho)^{n}\right) & 1-\frac{1-\pi}{1-\pi \rho}\left(1-(\pi \rho)^{n}\right)
\end{array}\right) .
$$




\subsection{Properties of $N(r)$}

In our first result we show how the pdf of $N(r)$ can be obtained recursively.

Lemma 3.1 Let $p_{r}(k)=P(N(r)=k)$ and $p_{r+1}(k)=P(N(r+1)=k)$. The following relation holds:

$$
\begin{aligned}
& p_{r+1}(0)=\pi p_{r}(0) \\
& p_{r+1}(k)=\pi p_{r}(k)+(1-\pi) \pi(1-\rho) \sum_{j=0}^{k-1} p_{r}(j) u^{k-j-1}, \quad k=1,2, \ldots,
\end{aligned}
$$

where $u=1-\pi(1-\rho)$.

Proof. Using the PGF, we see that $N(r+1) \stackrel{d}{=} N(r)+Y$. It follows that

$$
p_{r+1}(k)=\sum_{j=0}^{k} p_{r}(j) P(Y=k-j) .
$$

From here and using (2), it follows that $p_{r+1}(0)=p_{r}(0) \pi$ and for $k \geq 1$ that (8) holds.

Remark 3.1 If $\rho=0$, (8) can be simplified and we find $p_{r+1}(0)=\pi p_{r}(0)$ and

$$
p_{r+1}(k)=\pi \sum_{j=0}^{k} p_{r}(j)(1-\pi)^{k-j}, k=1,2, \ldots
$$

Using the PGF, we can also obtain the pdf of $N(r)$ explicitly. Taking $u=1-\pi(1-\rho)$, we find that

$$
\begin{aligned}
\psi_{N(r)}(t) & =\pi^{r}(1-\rho t)^{r} \frac{1}{(1-u t)^{r}} \\
& =\pi^{r} \sum_{k=0}^{r}\left(\begin{array}{l}
r \\
k
\end{array}\right)(-1)^{k} \rho^{k} t^{k} \sum_{i=0}^{\infty}\left(\begin{array}{c}
r+i-1 \\
i
\end{array}\right) u^{i} t^{i} .
\end{aligned}
$$

It follows that

$$
\psi_{N(r)}(t)=\pi^{r} \sum_{k=0}^{r} \sum_{j=k}^{\infty}\left(\begin{array}{l}
r \\
k
\end{array}\right)\left(\begin{array}{c}
r+j-k-1 \\
j-k
\end{array}\right)(-1)^{k} \rho^{k} u^{j-k} t^{j} .
$$

For $0 \leq n \leq r-1$, we find that

$$
\begin{aligned}
P(N(r)=n) & =\pi^{r} \sum_{k=0}^{n}\left(\begin{array}{l}
r \\
k
\end{array}\right)\left(\begin{array}{c}
r+n-k-1 \\
n-k
\end{array}\right)(-1)^{k} \rho^{k} u^{n-k} \\
& =\pi^{r} \sum_{i=0}^{n}\left(\begin{array}{c}
r \\
n-i
\end{array}\right)\left(\begin{array}{c}
r+i-1 \\
i
\end{array}\right)(-1)^{n-i} \rho^{n-i} u^{i}
\end{aligned}
$$


For $n \geq r$ we find that

$$
\begin{aligned}
P(N(r)=n) & =\pi^{r} \sum_{k=0}^{r}\left(\begin{array}{l}
r \\
k
\end{array}\right)\left(\begin{array}{c}
r+n-k-1 \\
n-k
\end{array}\right)(-1)^{k} \rho^{k} u^{n-k} \\
& =\pi^{r} \sum_{i=n-r}^{n}\left(\begin{array}{c}
r \\
n-i
\end{array}\right)\left(\begin{array}{c}
r+i-1 \\
i
\end{array}\right)(-1)^{n-i} \rho^{n-i} u^{i} .
\end{aligned}
$$

Summarizing, we have the following result.

Proposition 3.1 For the PMF of $N(r)$ we have

$$
P(N(r)=n)=\pi^{r} \sum_{i=\max (0, n-r)}^{n}\left(\begin{array}{c}
r \\
n-i
\end{array}\right)\left(\begin{array}{c}
r+i-1 \\
i
\end{array}\right)(-1)^{n-i} \rho^{n-i} u^{i} .
$$

A different approach to the PMF of $N(r)$ was studied in Minkova $(2001,2002)$. The PMF of $N(r)$ is given by

$$
\begin{aligned}
& P(N(r)=n) \\
& =\left\{\begin{array}{cl}
\pi^{r}, & n=0 \\
\pi^{r} \sum_{i=1}^{n}\left(\begin{array}{c}
n-1 \\
i-1
\end{array}\right)\left(\begin{array}{c}
r+i-1 \\
i
\end{array}\right)[(1-\pi)(1-\rho)]^{i} \rho^{n-i}, & n=1,2, \ldots
\end{array}\right.
\end{aligned}
$$

The proof of the next lemma is given in the Appendix.

Lemma 3.2 The PMF in (11) is equivalent to the PMF given in (12).

Remark 3.2 Note that for $\rho=0$, we have

$$
P(N(r)=n)=\left(\begin{array}{c}
r+n-1 \\
n
\end{array}\right) \pi^{r}(1-\pi)^{n}, \quad n \geq 0
$$

as expected.

Remark 3.3 Using the PGD of $N(r)$, we also have the following alternative representation. We have

$$
\begin{aligned}
\psi_{N(r)}(t) & =\left(\frac{1-\rho t}{1-\rho}\right)^{r}\left(\frac{\pi(1-\rho)}{1-(1-\pi(1-\rho)) t}\right)^{r} \\
& =\left(\frac{1}{1-\rho}+\frac{-\rho}{1-\rho} t\right)^{r}\left(\frac{\pi(1-\rho)}{1-(1-\pi(1-\rho)) t}\right)^{r} .
\end{aligned}
$$

If $\rho<0$, the first term is the PGF of a binomial r.v. $B(r) \sim B I N(r, \delta)$ with parameter $\delta=\frac{-\rho}{1-\rho}$. The second term is the PGF of an usual negative binomial r.v. $N^{*}(r)$ with parameter $\pi(1-\rho)$. We obtain that $N(r) \stackrel{d}{=} B(r)+N^{*}(r)$. This representation can be useful for simulation purposes. 
If $\rho>0$, we have

$$
\left(\frac{1-\rho}{1-\rho t}\right)^{r} \psi_{N(r)}(t)=\psi_{N^{*}(r)}(t)
$$

The first term on the left hand side corresponds to the PGF of an usual negative binomial r.v. $B^{*}(r)$ with parameter $\rho$. Now we obtain that $B^{*}(r)+N(r) \stackrel{d}{=} N^{*}(r)$.

Since $N(r)$ is the sum of i.i.d. random variables, we can apply the central limit theorem here.

Theorem 3.1 As $r \rightarrow \infty$, we have

$$
\frac{N(r)-r \mu}{\sqrt{r \sigma^{2}}} \stackrel{d}{\Longrightarrow} Z
$$

where $\mu$ and $\sigma^{2}$ are given in Section 2 and where $Z$ denotes a standard normal r.v.

Another limit theorem is given in the next result

Theorem 3.2 Suppose $r, \pi$ and $\rho$ vary in such a way that $r \rightarrow \infty, r(1-\pi) \rightarrow a>0$ and $\rho \rightarrow c \geq 0$. Then as $r \rightarrow \infty$, we have

$$
\psi_{N(r)}(t) \rightarrow \psi(t)=e^{-a(1-K(t))},
$$

where $K(t)=\frac{(1-c) t}{1-c t}$.

Proof. Let $u=1-\pi(1-\rho)$. We have

$$
\begin{aligned}
\psi_{N(r)}(t) & =\left(1-\left(1-\frac{\pi(1-\rho t)}{1-u t}\right)\right)^{r} \\
& =\left(1-\frac{r(1-\pi)(1-t)}{r(1-u t)}\right)^{r} \\
& \rightarrow \exp \left(-a\left(\frac{1-t}{1-c t}\right)\right),
\end{aligned}
$$

where we used the fact that $\pi \rightarrow 1$ and $u \rightarrow c$. Clearly the limit is of the form $e^{-a(1-K(t))}$, with $K(t)=\frac{(1-c) t}{1-c t}$.

The limit in the previous theorem has the following interpretation. Let $A, A_{1}, A_{2}, \ldots$ denote i.i.d. r.v. with the following geometric distribution:

$$
P(A=n)=(1-c) c^{n}, n=0,1, \ldots
$$

and let $A_{0}=0$. Also, let $V(a)$ denote a r.v. with a Poisson distribution with parameter $a$ :

$$
P(V(a)=n)=\frac{1}{n !} a^{n} e^{-a} .
$$

Using these notations, it follows that $\psi(t)$ is the PGF of the compound Poisson r.v. $N$, given by

$$
N=\sum_{0}^{V(a)} A_{i} .
$$




\section{The $\rho$ - Binomial distribution}

\subsection{Definition}

For $r \geq 1$, let $Y(r)=N(r)+r$ denote the number of experiments up to the $r^{t h}$ success and let $S_{n}$ denote the number of successes in the sequence $X_{1}, X_{2}, \ldots, X_{n}$. Clearly we have the following relations:

$$
\begin{aligned}
& P\left(S_{n} \geq r\right)=P(Y(r) \leq n) ; \\
& P(Y(r)=n)=P\left(S_{n}=r, X_{n}=1\right) .
\end{aligned}
$$

For $r=0$, we have $P\left(S_{n} \geq 0\right)=1$. We say that the r.v. $S_{n}$ has a binomial distribution related to the interrupted Markov chain with transition matrix $\mathbf{P}^{*}$. We call it the $\rho$-binomial distribution. Notation: $S_{n} \sim \operatorname{RHOBI}(n, \pi, \rho)$. Note that $S_{n}$ is the renewal process associated with $Y(r)$, i.e.,

$$
S_{n}=\sup \{r: Y(r) \leq n\}
$$

\subsection{Properties of $S_{n}$}

We clearly have

$$
\begin{aligned}
P\left(S_{n}=r\right) & =P(Y(r) \leq n)-P(Y(r+1) \leq n) \\
& =P(N(r) \leq n-r)-P(N(r+1) \leq n-r-1) .
\end{aligned}
$$

Let us denote $f_{S_{n}}(r)=P\left(S_{n}=r\right), r=0,1, \ldots, n$, the PMF of $S_{n}$.

Theorem 4.1 The PMF of $S_{n}$ is given by

$$
f_{S_{n}}(r)= \begin{cases}(1-\pi)[1-\pi(1-\rho)]^{n-1}, & r=0 \\
\pi^{r}+\pi^{r} \sum_{k=1}^{n-r} \sum_{i=1}^{k}\left(\begin{array}{c}
k-1 \\
i-1
\end{array}\right)\left(\begin{array}{c}
r+i-1 \\
i
\end{array}\right)[(1-\pi)(1-\rho)]^{i} \rho^{k-i} & \\
-\pi^{r+1}-\pi^{r+1} \sum_{k=1}^{n-r-1} \sum_{i=1}^{k}\left(\begin{array}{c}
k-1 \\
i-1
\end{array}\right)\left(\begin{array}{c}
r+i \\
i
\end{array}\right)[(1-\pi)(1-\rho)]^{i} \rho^{k-i}, & r=1, \ldots n-2, \\
\pi^{n-1}(1-\pi)[1+(n-1)(1-\rho)], & r=n-1 \\
\pi^{n}, & r=n .\end{cases}
$$


Proof. For $r=0$, we have

$$
\begin{aligned}
f_{S_{n}}(0) & =1-P(N(1) \leq n-1)=P(N(1)>n-1) \\
& =\pi \sum_{j=n}^{\infty} \sum_{i=1}^{j}\left(\begin{array}{l}
j-1 \\
i-1
\end{array}\right)[(1-\rho)(1-\pi)]^{i} \rho^{j-i} \\
& =(1-\pi)[1-\pi(1-\rho)]^{n-1} .
\end{aligned}
$$

For $r=1,2, \ldots n-2$, the proof follows from (13) and the PMF of $N(r)$ in (12). In the case of $r=n-1$, we have

$$
\begin{aligned}
f_{S_{n}}(n-1) & =P(N(n-1) \leq 1)-P(N(n) \leq 0) \\
& =\pi^{n-1}-\pi^{n-1}(n-1)(1-\pi)(1-\rho)-\pi^{n} . \\
& =\pi^{n-1}(1-\pi)[1+(n-1)(1-\rho)] .
\end{aligned}
$$

For $r=n,(13)$ gives $f_{S_{n}}(n)=P(N(n) \leq 0)=\pi^{n}$.

Information about the important moments of $S_{n}$ is given in the next Lemma, (Omey et al. (2008), Section 2.1).

Lemma 4.1 For $n \geq 1$, we have

$$
E\left(S_{n}\right)=n \frac{\pi(1-\rho)}{1-\pi \rho}+\frac{\rho \pi(1-\pi)}{(1-\pi \rho)^{2}}\left(1-(\rho \pi)^{n}\right)
$$

and

$$
\begin{aligned}
\operatorname{Var}\left(S_{n}\right) & =\frac{n \pi(1-\pi)(1-\rho)(1+\pi \rho)}{(1-\pi \rho)^{3}}+\frac{2 n(1-\pi) \pi \rho}{(1-\pi \rho)^{3}}\left[\pi(1-\rho)-(1-\pi)(\pi \rho)^{n}\right]+ \\
& +\frac{n^{2} \pi^{2}(1-\rho)^{2}}{(1-\pi \rho)^{2}}+\frac{(1-\pi) \pi \rho}{(1-\pi \rho)^{4}}\left[(1+\pi \rho)^{2}-2 \pi(1-\rho(1-\pi))\right]\left[1-(\pi \rho)^{n}\right] .
\end{aligned}
$$

Remark 4.1 The mean of the $\rho$ - binomial distribution is negatively dependent from the correlation coefficient. It follows from the fact that

$$
\frac{\partial}{\partial \rho} E S_{n}<0 .
$$

In the following theorem we give recursion formulas for PMF of the $\rho$ - binomial distribution. 
Theorem 4.2 The PMF of the $\rho$ - binomial distribution satisfies the following recursion:

$$
\begin{aligned}
& f_{S_{n}}(n)=p_{n}(0), \\
& f_{S_{n}}(r)=p_{r}(n-r)+(1-\pi) \sum_{j=0}^{n-r-1} p_{r}(j)(1-\pi(1-\rho))^{n-r-j-1}, r=1,2, \ldots n-1, \\
& f_{S_{n}}(0)=(1-\pi)[1-\pi(1-\rho)]^{n-1},
\end{aligned}
$$

where $p_{r}(0), p_{r}(1), \ldots p_{r}(n)$ are the probabilities of $I N B(\pi, \rho, r)$ distributed random variable, $r=0,1, \ldots n$.

Proof. The proof follows from (13) and the recursions (8) for the PMF of the INBD.

Remark 4.2 In the case of $\rho=0$ the formulas of Theorem 4.1 simplify to the usual binomial distribution, and we find

$$
\begin{aligned}
& f_{S_{n}}(n)=p_{n}(0), \\
& f_{S_{n}}(r)=p_{r}(n-r)+(1-\pi) \sum_{j=0}^{n-r-1} p_{r}(j)(1-\pi)^{n-r-j-1}, r=1,2, \ldots n-1, \\
& f_{S_{n}}(0)=(1-\pi)^{n},
\end{aligned}
$$

where $p_{r}(0), p_{r}(1), \ldots p_{r}(n)$ are the probabilities of $N B(\pi, r)$ distributed random variable, $r=0,1, \ldots n$.

\section{Concluding remarks}

Generalizations of the binomial distribution have been studied before. We mention, for example, the paper of Chang and Zelterman (2002). In this paper, the authors consider Bernoulli variables such that

$$
P\left(X_{n+1}=1 \mid X_{1}, X_{2}, \ldots, X_{n}\right)=P\left(X_{n+1}=1 \mid S_{n}\right),
$$

i.e., the success probability depends on the number of previous successes. In the paper of Drezner and Farnum (1993), the authors study Bernoulli variables such that

$$
P\left(X_{n+1}=1 \mid X_{1}, X_{2}, \ldots, X_{n}\right)=(1-\theta) p+\theta \frac{S_{n}}{n},
$$

for parameters $p$ and $\theta$.

In Omey et al. (2008), it is used a different approach in a general case. At first it is defined $S_{n}$, as the number of successes in the Markov sequence $X_{1}, X_{2}, \ldots$, and then the negative binomial r.v. $N(r)$ is defined by inverting of $S_{n}$.

In the case of interrupted Markov chain, the recursion formulas, given in Theorem 4.1, together with the recursions (8), could be used in numerical calculations. We plan to study further properties of the r.v. that we introduced in this paper. 


\section{Appendix}

\section{Proof of Lemma 3.2.}

Let us denote $v=(1-\rho)(1-\pi)$ such that $u=1-\pi(1-\rho)=v+\rho$. From $(9)$, for $n \leq r-1$, we obtain

$$
\begin{aligned}
P(N(r)=n) & =\pi^{r} \sum_{k=0}^{n}\left(\begin{array}{l}
r \\
k
\end{array}\right)\left(\begin{array}{c}
r+n-k-1 \\
n-k
\end{array}\right)(-1)^{k} \rho^{k}(v+\rho)^{n-k} \\
& =\pi^{r} \sum_{k=0}^{n}\left(\begin{array}{l}
r \\
k
\end{array}\right)\left(\begin{array}{c}
r+n-k-1 \\
n-k
\end{array}\right)(-1)^{k} \rho^{k} \sum_{j=0}^{n-k}\left(\begin{array}{c}
n-k \\
j
\end{array}\right) v^{j} \rho^{n-k-j} \\
& =\pi^{r} \sum_{j=0}^{n} v^{j} \rho^{n-j} \sum_{k=0}^{n-j}(-1)^{k}\left(\begin{array}{c}
r \\
k
\end{array}\right)\left(\begin{array}{c}
r+n-k-1 \\
n-k
\end{array}\right)\left(\begin{array}{c}
n-k \\
j
\end{array}\right) .
\end{aligned}
$$

From Prudnikov et al. (1986), it follows that for $j=0$

$$
\sum_{k=0}^{n}(-1)^{k}\left(\begin{array}{l}
r \\
k
\end{array}\right)\left(\begin{array}{c}
r+n-k-1 \\
n-k
\end{array}\right)=0 .
$$

For $j=1,2, \ldots$, we have

$$
\begin{aligned}
& \sum_{k=0}^{n-j}(-1)^{k}\left(\begin{array}{l}
r \\
k
\end{array}\right)\left(\begin{array}{c}
r+n-k-1 \\
n-k
\end{array}\right)\left(\begin{array}{c}
n-k \\
j
\end{array}\right) \\
& =\left(\begin{array}{c}
r+j-1 \\
j
\end{array}\right) \sum_{k=0}^{n-j}(-1)^{k}\left(\begin{array}{l}
r \\
k
\end{array}\right)\left(\begin{array}{c}
r+n-1-k \\
r+j-1
\end{array}\right) \\
& =\left(\begin{array}{c}
r+j-1 \\
j
\end{array}\right)\left(\begin{array}{c}
r+n-1-r \\
n-j
\end{array}\right)=\left(\begin{array}{c}
r+j-1 \\
j
\end{array}\right)\left(\begin{array}{c}
n-1 \\
j-1
\end{array}\right),
\end{aligned}
$$

where the last equation follows from Prudnikov et al. (1986), and then (A1) is equal to (12) for $n \leq r-1$.

Similarly, for $n=r, r+1, \ldots$, we have

$$
\begin{aligned}
P(N(r)=n) & =\pi^{r} \sum_{k=0}^{r}\left(\begin{array}{l}
r \\
k
\end{array}\right)\left(\begin{array}{c}
r+n-k-1 \\
n-k
\end{array}\right)(-1)^{k} \rho^{k} \sum_{j=0}^{n-k}\left(\begin{array}{c}
n-k \\
j
\end{array}\right) v^{j} \rho^{n-k-j} \\
& =\pi^{r} \sum_{j=0}^{n-r-1} v^{j} \rho^{n-j} \sum_{k=0}^{r}(-1)^{k}\left(\begin{array}{l}
r \\
k
\end{array}\right)\left(\begin{array}{c}
r+n-k-1 \\
n-k
\end{array}\right)\left(\begin{array}{c}
n-k \\
j
\end{array}\right) \\
& +\pi^{r} \sum_{j=n-r}^{n} v^{j} \rho^{n-j} \sum_{k=0}^{n-j}(-1)^{k}\left(\begin{array}{l}
r \\
k
\end{array}\right)\left(\begin{array}{c}
r+n-k-1 \\
n-k
\end{array}\right)\left(\begin{array}{c}
n-k \\
j
\end{array}\right) .
\end{aligned}
$$


So, for $j=0$ we obtain

$$
\sum_{k=0}^{r}(-1)^{k}\left(\begin{array}{l}
r \\
k
\end{array}\right)\left(\begin{array}{c}
r+n-k-1 \\
n-k
\end{array}\right)=\frac{r}{n} \sum_{k=0}^{r}(-1)^{k}\left(\begin{array}{l}
r \\
k
\end{array}\right)\left(\begin{array}{c}
r+n-1-k \\
n-1
\end{array}\right)=0 .
$$

For $j=1,2, \ldots$,

$$
\begin{aligned}
& \sum_{k=0}^{r}(-1)^{k}\left(\begin{array}{l}
r \\
k
\end{array}\right)\left(\begin{array}{c}
r+n-k-1 \\
n-k
\end{array}\right)\left(\begin{array}{c}
n-k \\
j
\end{array}\right) \\
& =\left(\begin{array}{c}
r+j-1 \\
j
\end{array}\right) \sum_{k=0}^{r}(-1)^{k}\left(\begin{array}{l}
r \\
k
\end{array}\right)\left(\begin{array}{c}
r+n-k-1 \\
n-k-j
\end{array}\right)=\left(\begin{array}{c}
r+j-1 \\
j
\end{array}\right)\left(\begin{array}{c}
n-1 \\
j-1
\end{array}\right) .
\end{aligned}
$$

Substituting (A4) and (A5) in (A3) leads to PMF (12) for $n=r, r+1, \ldots$.

\section{References}

Chang Y. and Zelterman D. (2002). Sums of dependent Bernoulli random variables and disease clustering, Stat. Probab. Lett., 57, 363 - 373.

Deng L - Y. and Chhikara R. S. (1990). On the characterization of the exponential distribution by the independence of its integer and fractional parts, Statistica Neerlandica, 44, $83-85$.

Drezner Z. and Farnum N. (1993). A Generalized Binomial Distribution, Commun. Statist. - Theory and Methods, 22, 3051 - 3063.

Feller W. (1971). An Introduction to Probability Theory and Its Applications, Vol.I, 2nd ed., John Wiley \& Sons, New York.

Johnson N. L., Kemp A. W. and Kotz S. (2005). Univariate Discrete Distributions, Third edition, John Wiley \& Sons, Hoboken, New Jersey.

Minkova L. D. (2001). A Family of Compound Discrete Distributions, Compt. Randue Bulg. Acad. Aci., 54, 11 - 14.

Minkova L. D. (2002). A Generalization of the Classical Discrete Distributions, Commun. Statist. - Theory and Methods, 31, 871 - 888.

Omey E. and Van Gulck S. (2006). Markovian Black and Scholes, Publications de l'institut mathèmatique, 79, 65 - 72 .

Omey E., Santos J. and Van Gulck S. (2008). A Markov - binomial distribution, Applicable Analysis and Discrete Mathematics, 2, 38 - 50.

Prudnikov A. P., Brychkov Yu. A. and Marichev O. I. (1986). Integrals and Series, vol. 1, Elementary Functions, Gordon \& Breach Sci. Publ., New York. 
Viveros R., Balasubramanian K. and Balakrishnan N. (1994). Binomial and Negative Binomial Analogues Under Correlated Bernoulli Trials, The American Statistician, 48, $234-247$. 